\title{
3 Research Suare \\ Did the COVID-19 pandemic stimulate telemedicine in pediatric diabetes care in Germany? A multicenter analysis of the year 2020 in the DPV-registry.
}

Klemens Raile ( $\sim$ klemens.raile@charite.de)

Charité - University Medicine Berlin

Torben Biester

Kinderkrankenhaus auf der Bult

Alexander Eckert

German Center for Diabetes Research

Simone von Sengbusch

University Hospital of Schleswig Holstein, Campus Lübeck

Anne Böhle

Catholic Children's Hospital Wilhelmstift

Uta Faller Faller

Oberschwabenklinik, Hospital St. Elisabeth, Department for Pediatrics

D Klee

Diabetological Practice, Bürstadt

Jessica Bokelmann

University Hospital of Schleswig Holstein, Campus Kiel

Joaquina Mirza

Kinderkrankenhaus Amsterdamerstrasse, Cologne

Reinhard Holl

Institute of Epidemiology and Medical Biometry, ZIBMT, University of Ulm

\section{Research Article}

Keywords: Telemedicine, type 1 diabetes, children, digital health, COVID-19, pandemia

Posted Date: March 3rd, 2022

DOI: https://doi.org/10.21203/rs.3.rs-1250023/v1

License: (c) (1) This work is licensed under a Creative Commons Attribution 4.0 International License.

Read Full License 


\section{Abstract}

Objectives: We analyzed uptake, volume and covariates of telemedicine use in children and adolescents with type 1 diabetes in 2020 with focus on the COVID-19 lockdown in Germany and provide first outcome data associated with telemedicine use.

Research design and methods: Based on data from the German Diabetes Prospective Follow-up Registry (DPV) in 2020, we included 247 pediatric diabetes centers following 26,365 children and adolescents with type 1 diabetes. Centers and individuals were characterized according to telemedicine use. Effects of the lockdown from March $13^{\text {th }}$ to May $13^{\text {th }} 2020$ versus the entire years 2020 and 2019 were analyzed.

Results: During the lockdown in 2020, telemedicine visits increased from pre-lockdown $0.6 \%$ of all patient visits to $12.8 \%$ and remained $3.5 \%$ until end of 2020 . Among 247 centers only 11 provided telemedicine to more than 50 patients, while telephone contacts were the preferred telemedicine-mode during lockdown. Patients receiving telemedicine in 2020 had lower HbA1c, longer distance to diabetes centers, less frequently migration background and higher use of insulin pumps or CGMS.

Discussion: Telemedicine experienced a dramatic boost during and after the strict lockdown in Germany. Anyhow absolute numbers of telemedicine in outpatient contacts remained below $5 \%$ and thus telemedicine did not take a load-carrying role in pediatric diabetes care in Germany. Having migration background and not using diabetes technology possibly reflect barriers to scale up digital communication.

\section{Introduction}

Coronavirus disease 2019 (COVID-19) and the subsequent social distancing rose to a global public health emergency, challenging health systems worldwide and forcing the rapid adoption of any preexisting telemedicine strategies [1]. In Germany, a dramatic increase in diabetic ketoacidosis and severe ketoacidosis in children with new-onset type 1 diabetes (T1DM) happened during the first lock-down, while the incidence appeared to remain stable $[2,3]$. This indicated several barriers to diagnose new-onset diabetes in children early, but also to access medical care timely in the COVID-lockdown situation.

Type 1 diabetes in children and adolescents is a highly relevant field for remote medical care, with modern devices such as insulin pumps and continuous glucose-monitoring (CGM) having been established in more than $50 \%$ of all individuals in Germany / Austria and in the US. Many of these systems deliver patient-derived health data into data platforms and analysis software mostly as part of cloud-based manufacturers' ecosystems. Therefore pediatric diabetology takes a pioneer position for telemedicine both in pediatrics and in diabetology $[4,5]$. In general, these tools, if established, enabled immediate distancing of any existing in-person diabetes care during the COVID-19 pandemic [6, 7]. International pediatric guidelines also describe technology as an important factor for outpatient care and recommend stricter glycemic targets when these technical devices, embedded into a comprehensive care concept, are used [8]. The majority of patients, caretakers and health care professionals were already 
familiar with these systems. However, in Germany, organizational framework and hardware availability for video consultations to be used during lockdown conditions was available only in few studies or centers $[1,9]$ and implementation of a complete telemedicine eco-environment was new for regular patient care to most health care providers. Multiple stakeholders are involved and preexisting regulations make the practical implementation time consuming and challenging [10,11]. Metabolic outcome data, how telemedicine helped to manage diabetes care for children and adolescents during the COVID-19pandemic, were already published from European centers $[12,13]$. The rapid adoption of telemedicine in the US during the COVID-19 pandemic was already reported from the T1DX-QI but comparison to larger T1D surveys in Europe were still lacking [14]. In detail, we analyze here (i) whether and how such digital infrastructures were activated to perform digital health care for children and youth during the COVIDpandemic, (ii) what are center characteristics providing telemedicine (iii) what are specific clinical characteristics of patients experiencing telemedicine follow ups. We also address the optimistic view that the experience gained from emergency care during the pandemic has already boosted the use of telemedicine and telemonitoring [15] - at least in Germany.

\section{Research Design And Methods}

The DPV Registry is a long-term surveillance tool of patients with diabetes in Germany, Austria, Luxembourg and Switzerland, also collecting detailed data on use of CGM and insulin pumps $[8,16]$. DPV covers more than $90 \%$ of all pediatric individuals with T1D and provides representative data reflecting a real-world scenario of all aspects of diabetes management in Germany [17].

We analyzed the complete 2020 DPV data report from 26,365 German children and adolescents with T1D being followed at their respective diabetes centers and having follow-up data in 2020. Overall 2,623 of them $(9.6 \%)$ had at least one telemedicine contact in 2020 . If data were available, we depict diabetes care specifically during the COVID-19-lockdown from March 13th to May 13th 2020 in Germany with all medical and psychosocial data. In DPV, telemedicine contacts could be entered directly either as "video", "telephone" or "email". If both "email" and "telephone" were documented for one visit, type of telemedicine was considered as "telephone + email". An email contact alone was not sufficient to be categorized as telemedicine. If any type of telemedicine was documented we considered this contact as telemedicine, even if also other inpatient or outpatient contacts happened in the same acquisition period. Our search was completed by a string search in open text fields with the terms "tele", "video", "email", "online", "digital" and "vidiki". "Vidiki" is the largest prospective telemedicine project in pediatric diabetology in Germany [18]. Consent was obtained from patients or caretakers. The ethics committee of the University of Ulm approved scientific analysis of anonymized data, and institutional review boards approved data collection in local institutions. Informed consent was obtained from all participants and/or their legal guardians in local institutions. All methods were carried out in accordance with relevant guidelines and regulations.

We compared TM use from January 1st 2020 until December 31th 2020 with identical, seasonal intervals in 2019 to see changes induced by the first COVID-19 lockdown phase in Germany. Patient contacts were 
classified as outpatient (ambulatory), inpatient (hospital admission) or telemedicine. Frequencies were assessed per week. The centers providing TM were characterized by size of center (number of patients), by the categories university versus community hospital, by mean airline distance between patient's home and the hospital (\% of patients living $\geq 25 \mathrm{~km}$ away from the center) and the mode of TM used (video, telephone, email, undefined). The patients were described by age, gender, diabetes duration, mode of insulin therapy, hemoglobin $\mathrm{A} 1 \mathrm{c}$ ( $\mathrm{HbA1c}$, standardized to DCCT reference range), migration background (at least one parent being born outside of Germany), CGM-use and time-in-range, as well as airline distance to their specific diabetes center (see above). Description was conducted for the entire cohort and stratified by TM use within the respective time window.

Statistics were calculated using SAS (Statistical Analysis Software, SAS Institute Inc., Cary, NC, USA) Version 9.4 build TS1M7 on a windows 2019 server mainframe. Descriptive statistics was performed for base-line characteristics of our cohort (Table 2). The results were shown as median with quartiles for continuous variables using the Kruskal-Wallis-Test to compute unadjusted $p$-values for group comparison, and as proportions for binary variables, using the $\chi^{2}$-test for comparison.

\section{Results}

Pediatric diabetes centers providing telemedicine. We characterized the centers that provided telemedicine in 2020. Out of 247 pediatric diabetes centers that contributed data of overall 26,365 patients, 97 documented any use of telemedicine in 2,623 of their patients. But approximately one third (36 centers) only sporadically documented single patient-contacts ( 5 or less) via any kind of telemedicine communication. Important to note, regular calls for prescriptions or to postpone appointments were not counted as "telemedicine" contact. Anyhow, only 11 centers provided telemedicine care for more than 50 patients or in average more than $20 \%$ of their respective patients in routine care (Table 1 ) and only single centers had established video consultations at larger numbers for their patients (Fig. 1). Moreover, those 61 centers providing more than 5 annual telemedicine contacts in 2020 had a larger share of patients living $>25 \mathrm{~km}$ away from respective diabetes centers (28.6\% versus $24.1 \%$ ) and a higher prevalence of university clinics (29.5\% versus $11.8 \%)$. Interestingly, only 24 of those 61 centers used telemedicine before the lockdown, another 29 centers started in the lockdown and a final number of 8 centers had first virtual contacts after end of lockdown in 2020. 
Table 1

All 247 pediatric DPV centers and their patients followed completely or intermittently (once or more) with telemedicine (TM) in 2020.

\begin{tabular}{|lllll|}
\hline Patients treated via TM per center & Centers & Patients in 2020 & TM & \% TM \\
\hline No TM & 150 & 10,771 & 0 & $0 \%$ \\
\hline $1-5$ & 36 & 4,101 & 69 & $1.6 \%$ \\
\hline $6-10$ & 11 & 1,634 & 84 & $5.0 \%$ \\
\hline $11-25$ & 23 & 3,155 & 392 & $12.1 \%$ \\
\hline $26-50$ & 16 & 2,977 & 567 & $18.7 \%$ \\
\hline $51-100$ & 6 & 1,483 & 391 & $25.7 \%$ \\
\hline$>100$ & 5 & 2,244 & 1,120 & $48.7 \%$ \\
\hline Total & 247 & 26,365 & 2,623 & $9.6 \%$ \\
\hline
\end{tabular}

Medical contacts in 2020. In the entire year 2020, 102,514 medical contacts of 26,365 children and adolescents with T1D were documented (visualized in Fig. 2). In detail 88,684 outpatient visits (86.5\%), 9,701 hospital admissions (9.5\%) and 4,129 telemedicine contacts (4.0\%). Most of those 26,365 children experienced outpatient contacts alone, and if telemedicine was involved, as a mixture of all types of medical contacts, predominantly outpatient plus telemedicine contacts. Telemedicine alone was limited to single individuals (Table 2). Telemedicine was further specified as "telephone contacts", "telephone and emails", "video consultations" and finally others that had not been further specified (Table 3). Interestingly, video consultations had not been established in the majority of these contacts, reflecting the telemedicine techniques provided by the centers. Within the strict lockdown period between March 13th and May 13th, the total number of in-person visits decreased compared to the same interval in 2019 both for outpatient visits from 88.5-78.4\% $(p<0.001)$ and inpatient admissions from 11.0-8.6\% $(p<0.001)$. During this period, telemedicine visits increased from pre-lockdown $0.6 \%$ of all patient visits to $12.8 \%$ and remained 3.5\% until end of 2020. Pre-lockdown was similar to the same period in 2019 (0.5\%, data given in table S1 and depicted in Figure S2) and thus lockdown-conditions stimulated higher use of telemedicine. 
Table 2

All types of contacts and possible combinations within the entire year 2020 (left) and exclusively during the lockdown period (right).

\section{Entire year 2020 \\ Lockdown March 13th - May 13th 2020}

Type of contact

Patient numbers (\% of Patient numbers (\% of all)* all)

\begin{tabular}{lll} 
Inpatient & $1,062(4.0 \%)$ & $616(4.1 \%)$ \\
\hline Outpatient & $16,476(62.5 \%)$ & $8,749(58.3 \%)$ \\
\hline Telemedicine & $19(0.1 \%)$ & $1,056(7.0 \%)$ \\
\hline Inpatient + outpatient & $6,204(23.5 \%)$ & $460(3.1 \%)$ \\
\hline Inpatient + telemedicine & $25(0.1 \%)$ & $36(0.2 \%)$ \\
\hline Outpatient + telemedicine & $1,702(6.5 \%)$ & $414(2.8 \%)$ \\
\hline $\begin{array}{l}\text { Inpatient + outpatient + } \\
\text { telemedicine }\end{array}$ & $877(3.3 \%)$ & $23(0.2 \%)$ \\
\hline Total & $26,365(100.0 \%)$ & $11,354(100.0 \%)$
\end{tabular}

* Percent (\%) refers to those 11,354 patients that had any contact to their diabetes centers, the remaining 15,011 patients had no contacts within the lockdown period.

Table 3

Spectrum of telemedicine (TM) contacts within the entire year 2020 (left) or exclusively during the lockdown period (right).

\begin{tabular}{|lll|}
\hline & Entire year 2020 & Lockdown March 13th - May 13th 2020 \\
\hline Type of telemedicine & Contacts (\% of all) & Contacts (\% of all) \\
\hline Telefone & $1641(39.7 \%)$ & $890(52.2 \%)$ \\
\hline Telephone+ email & $229(5.5 \%)$ & $89(5.2 \%)$ \\
\hline Video & $1225(29.7 \%)$ & $404(23.7 \%)$ \\
Not specified & $1034(25.0 \%)$ & $322(18.9 \%)$ \\
\hline Total & $4129(100.0 \%)$ & $1705(100.0 \%)$ \\
\hline
\end{tabular}

Patients experiencing telemedicine in 2020. As shown above, most individuals who experienced telemedicine contacts used those interchangeable with in-person and outpatient contacts (Table 2) and thus telemedicine helped to bridge continuous diabetes follow up. When compared to those not experiencing telemedicine, telemedicine users lived further away from their respective diabetes center, used more insulin pump and CGM technology or were less frequent from migration background (Table 4, left). HbA1c, gender, diabetes duration and BMI did not differ between both groups (Table 4, left). In a 
smaller cohort of 2,341 patients that had regular CGM-downloads into DPV in 2020, time-in-range was slightly higher in those also using telemedicine.

Table 4

Demographics and clinical data of all 26,365 patients followed intermittently with telemedicine (TM, $n=$ 2,623 ) or completely without telemedicine (no TM, $n=23,742$ ) over the entire year 2020 (left) and of 11,354 patients analyzed during the lockdown period (March 13th to May 13th 2020.

\begin{tabular}{|c|c|c|c|c|c|c|}
\hline \multirow[t]{2}{*}{ Period } & \multicolumn{3}{|c|}{$\begin{array}{l}\text { January - December } 2020 \\
\text { (including lockdown-period) }\end{array}$} & \multicolumn{3}{|c|}{$\begin{array}{l}\text { Only lockdown-period - March 13th - } \\
\text { May 13th } 2020 .\end{array}$} \\
\hline & $\begin{array}{l}\text { No } \\
\text { telemedicine }\end{array}$ & Telemedicine & $P$ & $\begin{array}{l}\text { No } \\
\text { telemedicine }\end{array}$ & Telemedicine & $P$ \\
\hline$N$ & 23,742 & 2,623 & n.a. & 9,831 & 1,523 & n.a. \\
\hline Age [y] & $\begin{array}{l}13.1(9.7- \\
15.7)\end{array}$ & $\begin{array}{l}12.5(9.0- \\
15.3)\end{array}$ & $<.001$ & $\begin{array}{l}12.9(9.5- \\
15.5)\end{array}$ & $\begin{array}{l}12.8(9.5- \\
15.4)\end{array}$ & n.s. \\
\hline Male [\%] & 53.2 & 52.3 & n.s. & 53 & 54 & n.s. \\
\hline $\begin{array}{l}\text { Migration backgr. } \\
{[\%]}\end{array}$ & 26.2 & 20.1 & $<0.001$ & 28.5 & 19.8 & $<.001$ \\
\hline $\begin{array}{l}\text { Diabetes duration } \\
\text { [y] }\end{array}$ & $\begin{array}{l}4.0(1.5- \\
7.4)\end{array}$ & $3.7(1.4-7.2)$ & n.s. & $\begin{array}{l}3.9(1.5- \\
7.1)\end{array}$ & $4.1(1.6-7.5)$ & 0.25 \\
\hline BMI SDS & $\begin{array}{l}0.35(-0.3- \\
1.0)\end{array}$ & $\begin{array}{l}0.34(-0.3- \\
9.7)\end{array}$ & n.s. & $\begin{array}{l}0.36(-0.26- \\
1.00)\end{array}$ & $\begin{array}{l}0.31(-0.26- \\
0.94)\end{array}$ & n.s. \\
\hline HbA1c [\%] & $\begin{array}{l}7.7(7.0- \\
8.6)\end{array}$ & $7.6(7.0-8.4)$ & n.s. & $\begin{array}{l}7.7(7.0- \\
8.6)\end{array}$ & $8.0(7.2-9.1)$ & 0.006 \\
\hline $\begin{array}{l}\mathrm{HbA} 1 \mathrm{c} \\
{[\mathrm{mmol} / \mathrm{mol} \mathrm{Hb}]}\end{array}$ & $\begin{array}{l}60.2(53.0- \\
70.0)\end{array}$ & $\begin{array}{l}59.9(52.7- \\
68.7)\end{array}$ & n.s. & $\begin{array}{l}60.4(52.5- \\
70.4)\end{array}$ & $\begin{array}{l}64.0(55.2- \\
75.8)\end{array}$ & 0.006 \\
\hline $\begin{array}{l}\text { TDD insulin [U/kg } \\
\text { BW/d] }\end{array}$ & $\begin{array}{l}0.82(0.64- \\
1.03)\end{array}$ & $\begin{array}{l}0.79(0.62- \\
1.00)\end{array}$ & $<.001$ & $\begin{array}{l}0.83(0.65- \\
1.05)\end{array}$ & $\begin{array}{l}0.78(0.61- \\
1.03)\end{array}$ & 0.032 \\
\hline CSII [\%] & 56.8 & 61.0 & $<001$ & 55.6 & 57.1 & n.s. \\
\hline §CGM use [\%] & 79.8 & 88.0 & $<.001$ & n.d. & n.d. & \\
\hline $\begin{array}{l}\text { Distance to } \\
\text { center }>25 \mathrm{~km} \text { [\%] }\end{array}$ & 24.8 & 27.9 & $\begin{array}{l}= \\
0.006\end{array}$ & 22.6 & 28.9 & $\begin{array}{l}< \\
0.001\end{array}$ \\
\hline \#TIR [\%] & $\begin{array}{l}54.1(41.9- \\
66.3)\end{array}$ & $\begin{array}{l}57.2(46.3- \\
69.2)\end{array}$ & $<.001$ & n.d. & n.d. & \\
\hline $\begin{array}{l}\text { CSII: insulin pump } \\
\text { of insulin; CGM: Co } \\
\text { entire year 2020. } \\
\text { were shown as mec }\end{array}$ & apy; BMI SD & $\begin{array}{l}\text {, data from } 2,3 \\
\text { artile range. }\end{array}$ & stand & d deviation sc & e; TDD: total da & $\begin{array}{l}\text { dose } \\
\text { rthe } \\
\text { ata }\end{array}$ \\
\hline
\end{tabular}


Focusing on the strict lockdown period in Germany from March 13th to May 13th (Table 4, right) migration background and distance to the diabetes center above $25 \mathrm{~km}$ were higher in those with telemedicine visits. But in contrast to the entire year 2020, HbA1c in those with TM-contacts was specifically found higher in this sub-cohort. But important to note, the majority of 15,011 patients had no medical contacts in the strict lockdown period.

\section{Discussion}

We were interested to follow implementation of telemedicine care in children and adolescents with T1D in Germany and particularly focused on whether telemedicine worked as ad hoc uptake during the strict COVID-19-lockdown period, as it has been strongly demonstrated by the T1DX-QI register [14]. The DPV registry prospectively surveys more than $90 \%$ of all individuals with T1D younger than 18 years in Germany. Here we give a detailed picture of first, patients that had a chance to use telemedicine in 2020 with special focus on the strict lockdown weeks in Germany, and second, on those diabetes centers that established some level of telemedicine compared to centers who provided in-person visits only.

In Germany, most children and adolescents with T1D receive continuous outpatient care in specialized pediatric diabetes centers providing both inpatient and outpatient care. The "COVID-19-lockdown" was imposed all over Germany and within days decisions were made on what kind of in-person visits needed to be classified as "urgent" or had to be cancelled. Any type of telemedicine was immediately used to contact families and assure that prescriptions for devices, supplies and insulin were sufficient. All routine contacts should be postponed or switched to telemedicine contacts, if accepted by the families.

Interestingly, even under this conditions, the majority of outpatient contacts were postponed over the twomonth-period or still continued as in-person visits, while telemedicine visits rose to $12.8 . \%$. This was remarkably higher than $0.5 \%$ in the same period of 2019 but far from the $95 \%$ rise in telemedicine visits found in the T1DX-QI for the US [14]. Our evaluation revealed that only 61 centers used telemedicine in more than 5 of their patients and their mode of telemedicine ranged from almost exclusively using video consultation to primarily using telephone calls. Telephone calls were also the major strategy for telemedicine contacts with families and patients during the lockdown weeks. As reported above, the majority of the 61 centers started documenting telemedicine visits during lockdown until May 13th, so that COVID-lockdown clearly fueled onset of telemedicine in most of those centers.

Remarkably, in-person contacts were significantly reduced during the lockdown phase, compared to identical calendar weeks in 2019. But the decrease to $78.6 \%$ compared to $88.5 \%$ in 2019 was moderate and obviously, the majority of pediatric diabetes appointments had been classified as "urgent medical", not allowing to be postponed along with local lockdown regulations. Hospital admissions dropped and were also restricted to acute diabetes complications. Telemedicine contacts increased, but their absolute numbers were not sufficient to compensate for all of the postponed in-person appointments. Also only a minority of pediatric diabetes centers in Germany were prepared and could provide any of the telemedicine options as the lockdown suddenly impacted on diabetes care. 
The COVID-19-pandemic and in particular lockdown regulations should catalyze telemedicine care of children and adolescents with T1D and some authors even emphasized a paradigm change in pediatric diabetes care by the pandemic [15]. The majority of children and adolescents treated in pediatric centers in Germany use a CGMS and insulin pump [4] and thus already had technical prerequisites to upload and share their glucose values and insulin-treatment data. We also found that longer travelling distance to diabetes centers favored but migration background of families restricted the use of telemedicine. This second aspect points to language and other socioeconomic factors that could impair any nondiscriminatory and fair expansion of telemedicine use.

We found no deterioration of metabolic control over the lockdown weeks, at least in terms of HbA1c and time-in-range, irrespective of whether contacts were switched to telemedicine or kept in-person. In Sweden, the results during the first 7 months (Jan-July 2020) of the pandemic showed no deterioration of $\mathrm{HbA1c}$, blood lipids and time-in-range, compared to corresponding periods within previous years.[13] These medical data give us some relief, but we should interpret with care, how the Corona pandemic and in particular what adaptations of treatment policies may have impacted on T1D care. Telemedicine usability might only be one of many changes resulting from the pandemic [19].

Centers' perspective (Fig. 1) gave a heterogeneous picture of individual centers in Germany, even if a similar number of patients were followed by telemedicine. Some centers used video consultations, others telephone calls in almost identical frequencies, but only a minority of 11 centers managed to follow more than 50 patients per year via telemedicine. Looking at all German patients in DPV and their caretakers, less than $10 \%$ experienced any telemedicine contact in 2020 and as mentioned above only few centers provided telemedicine on a regular base. Thus, telemedicine in 2020 was already a light at the end of the tunnel but not a load-bearing infrastructure for pediatric diabetes care in Germany.

Looking ahead, some barriers to scale up telemedicine in Germany are identified so far. First, the European Union enacted the General Data Protection Regulation (GDPR) and up to now, some manufacturers' software solutions are still not completely GDPR compliant and local hospital data protection regulations blocked active upload of patient data into these data-sharing cloud software systems. Second, implementation of video consultations into hospital ecosystems is a long process for health care professionals and requires investments into hardware, software and upload devices. However, a qualitative assessment of diabetes educators and pediatric diabetologists who were familiar with telemedicine found that video consultation offered several benefits in the ongoing care of children with T1D [20].

In conclusion, we experienced an impressive dynamics of telemedicine within the COVID-lockdown phase in the US but also in Germany, and finally use of telemedicine established at a much higher rate thereafter. This reflects very good acceptance once telemedicine was established - not only under emergency conditions. However, the overall use of telemedicine in Germany was unable to compensate any reduction of in-person visits and has not been established in the majority of our pediatric diabetes centers. Additional key factors to scale up telemedicine for children with diabetes in Germany were (i) 
GDPR compliant telehealth or video consultation programs and associated health data platforms, (ii) addressing specific socioeconomic demands and (iii) securing equal financing of telemedicine compared to in-person visits.

\section{Declarations}

\section{Data availability}

To protect patient privacy, patient level data cannot be shared with outside investigators. However, upon request and after agreement from the DPV scientific board, joint research projects based on remote data analysis are possible. These requests should be addressed to the email of the last author: reinhard.hol/@uni-ulm.de.

\section{Acknowledgements}

This work was partly funded by the Deutsche Diabetes Stiftung (FP-0433-2020)

We wish to thank all centres participating in the DPV project, as well as all patients donating their data to the DPV registry. For a full list of participating DPV centres, see the supplement. Special thanks to $A$. Hungele and R. Ranz for development of the DPV documentation software and K. Fink and E. Bollow for the DPV data management (clinical data managers, Ulm University). This work was supported by the German Centre of Diabetes Research (DZD) funded by the Federal Ministry of Education and Research (FKZ 82DZD14E03). Further financial support for the DPV registry was provided by the German Diabetes Association (DDG 2020) and the Robert Koch Institute (RKI) diabetes-surveillance 1368-1711.

\section{Author contributions}

$K R$ and TB designed the trial, the concept and data to be included was decided in video conference by the whole author's board. Statistical analysis was performed by AE und RWH. RWH coordinates the DPV initiative and conceptualized the research projects on COVID effects on pediatric diabetes care. The first draft of the manuscript was written by TB and KR, edited by RWH and SVS, read, re-edited and acknowledged by all authors.

\section{Competing Interests Statement}

$\mathrm{KR}$ is AB-member of Lilly Diabetes Care and Abbott Diabetes Care, received speakers honorary from Novonordisk, Sanofi and Lilly, and has a collaborative incentive with Dexcom. TB received speaker's honoraria from AstraZeneca, DexCom, Medtronic, NovoNordisk, Roche, Sanofi. SVS received speaker's honoraria from Abbott, Berlin-Chemie, Dexcom Infectopharm, Lilly, Medtronic, NovoNordisk and Sanofi and reports being a consultant for Abbott, Lilly, Medtronic and NovoNordisk. JB received speaker's honoria from Mediq Direkt, Lilly, Pfizer, Merck Serono and RG Ärztefortbildung. AB, AE, DK JM, RWH and UF reports no conflict of interest. 


\section{References}

1. Braune K, Boss K, Schmidt-Herzel J, et al. (2020) "Shaping Workflows in Digital and Remote Diabetes Care During the COVID-19 Pandemic: A Service Design Approach." JMIR Mhealth Uhealth.

2. Tittel SR, Rosenbauer J, Kamrath C, et al. (2020) "Did the COVID-19 Lockdown Affect the Incidence of Pediatric Type 1 Diabetes in Germany?" Diabetes Care 43(11): e172-e173.

3. Kamrath C, Monkemoller K, Biester T, et al. (2020) "Ketoacidosis in Children and Adolescents With Newly Diagnosed Type 1 Diabetes During the COVID-19 Pandemic in Germany." JAMA 324(8): 801804.

4. van den Boom L, Karges B, Auzanneau M, et al. (2019) "Temporal Trends and Contemporary Use of Insulin Pump Therapy and Glucose Monitoring Among Children, Adolescents, and Adults With Type 1 Diabetes Between 1995 and 2017." Diabetes Care 42(11): 2050-2056.

5. Miller KM, Hermann J, Foster N, et al. (2020) "Longitudinal Changes in Continuous Glucose Monitoring Use Among Individuals With Type 1 Diabetes: International Comparison in the German and Austrian DPV and U.S. T1D Exchange Registries." Diabetes Care 43(1): e1-e2.

6. Garg SK, Rodbard D, Hirsch IB and Forlenza GP (2020) "Managing New-Onset Type 1 Diabetes During the COVID-19 Pandemic: Challenges and Opportunities." Diabetes Technol Ther 22(6): 431-439.

7. Peters AL and Garg SK (2020) "The Silver Lining to COVID-19: Avoiding Diabetic Ketoacidosis Admissions with Telehealth." Diabetes Technol Ther 22(6): 449-453.

8. Sherr JL, Tauschmann M, Battelino T, et al. (2018) "ISPAD Clinical Practice Consensus Guidelines 2018: Diabetes technologies." Pediatr Diabetes 19 Suppl 27: 302-325.

9. von Sengbusch S, Eisemann N, Mueller-Godeffroy E, et al. (2020) "Outcomes of monthly video consultations as an add-on to regular care for children with type 1 diabetes: A 6-month quasirandomized clinical trial followed by an extension phase." Pediatr Diabetes 21(8): 1502-1515.

10. Jones MS, Goley AL, Alexander BE, et al. (2020) "Inpatient Transition to Virtual Care During COVID-19 Pandemic." Diabetes Technol Ther 22(6): 444-448.

11. March CA, Flint A, DeArment D, et al. (2020) "Paediatric diabetes care during the COVID-19 pandemic: Lessons learned in scaling up telemedicine services." Endocrinol Diabetes Metab: e00202.

12. Tornese G, Schiaffini R, Mozzillo E, et al. (2021) "Telemedicine in the Time of the COVID-19 Pandemic: Results from the First Survey among Italian Pediatric Diabetes Centers." Healthcare (Basel) 9(7).

13. Ludvigsson J (2021) "Effect of COVID-19 pandemic on treatment of Type 1 diabetes in children." Acta Paediatr 110(3): 933-934.

14. Lee JM, Carlson E, Albanese-O'Neill A, et al. (2021) "Adoption of Telemedicine for Type 1 Diabetes Care During the COVID-19 Pandemic." Diabetes Technol Ther 23(9): 642-651.

15. Danne T, Limbert C, Puig Domingo M, et al. (2021) "Telemonitoring, Telemedicine and Time in Range During the Pandemic: Paradigm Change for Diabetes Risk Management in the Post-COVID Future." Diabetes Ther 12(9): 2289-2310. 
16. Tauschmann M, Hermann JM, Freiberg C, et al. (2020) "Reduction in Diabetic Ketoacidosis and Severe Hypoglycemia in Pediatric Type 1 Diabetes During the First Year of Continuous Glucose Monitoring: A Multicenter Analysis of 3,553 Subjects From the DPV Registry." Diabetes Care 43(3): e40-e42.

17. Addala A, Auzanneau M, Miller K, et al. (2021) "A Decade of Disparities in Diabetes Technology Use and HbA1c in Pediatric Type 1 Diabetes: A Transatlantic Comparison." Diabetes Care 44(1): 133140.

18. Frielitz FS, Muller-Godeffroy E, Hubner J, et al. (2020) "Monthly Video-Consultation for Children With Type 1 Diabetes Using a Continuous Glucose Monitoring System: Design of ViDiKi, a Multimethod Intervention Study to Evaluate the Benefit of Telemedicine." J Diabetes Sci Technol 14(1): 105-111.

19. Prabhu Navis J, Leelarathna L, Mubita W, et al. (2021) "Impact of COVID-19 lockdown on flash and real-time glucose sensor users with type 1 diabetes in England." Acta Diabetol 58(2): 231-237.

20. Frielitz FS, Dordelmann J, Lemke S, et al. (2021) "Assessing the benefits and challenges of video consultations for the treatment of children with type 1 diabetes - A qualitative study among diabetes professionals." Exp Clin Endocrinol Diabetes 129(11):831-836.

\section{Figures}

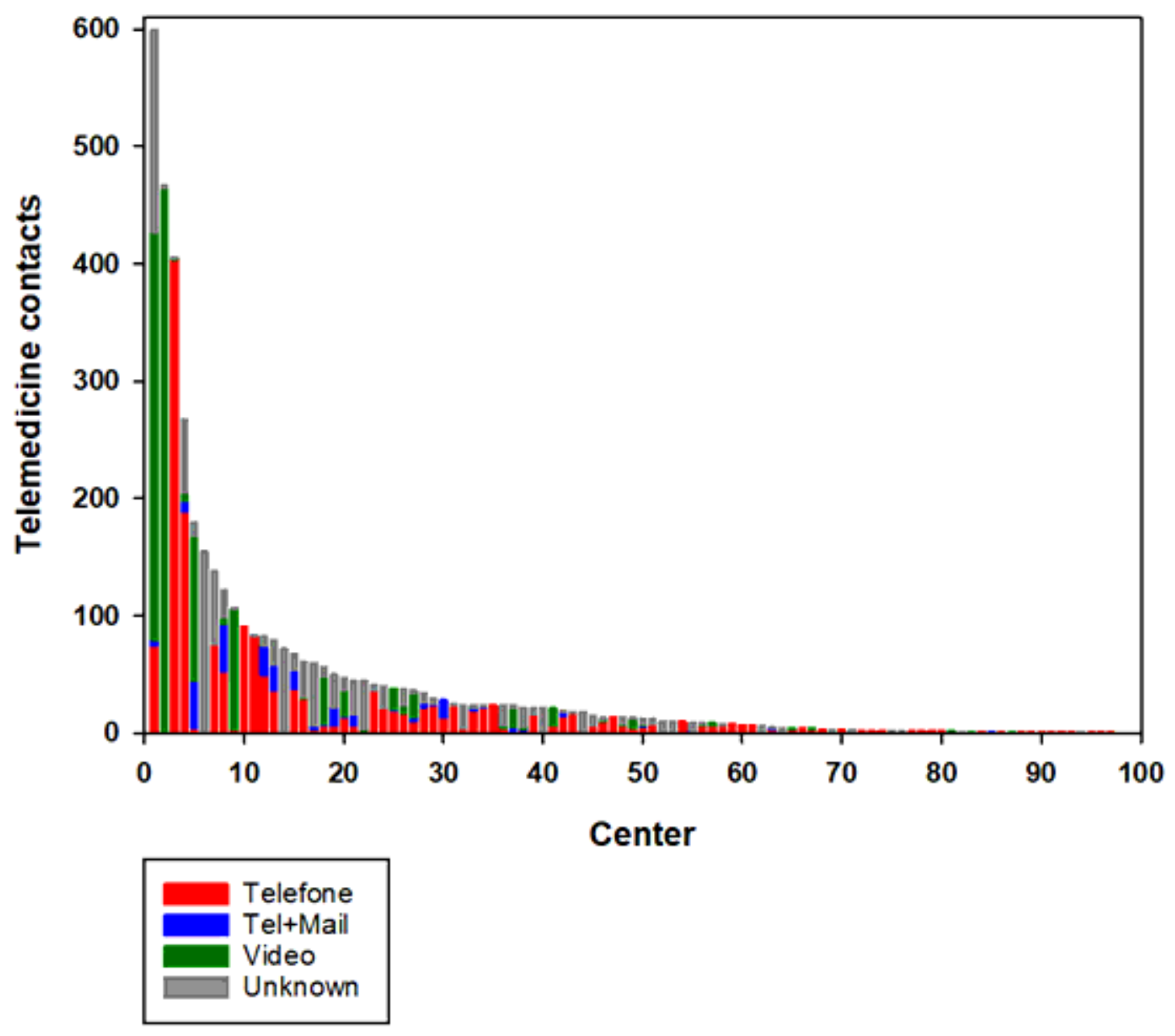


Figure 1

Center perspective of all 97 DPV centers providing any type of telemedicine (corresponding to table 1). Only 11 centers documented more than 50 TM contacts in 2020, video consultations and telephone were predominantely used in the four largest centers.

\section{Lockdown}

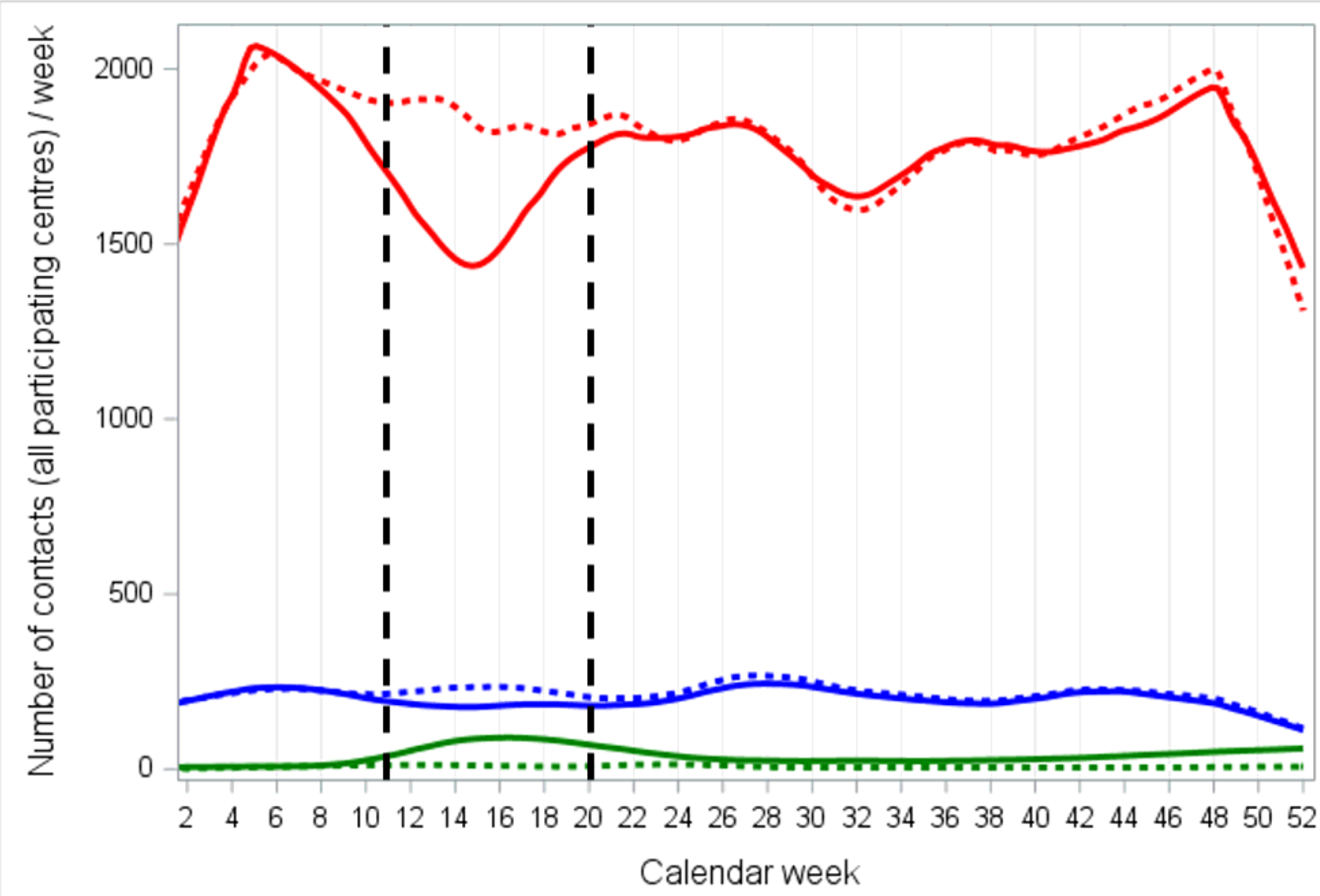

Telemedicine 2020

Inpatient 2020

Outpatient 2020

..... Telemedicine 2019

...... Inpatient 2019

-...-O Outpatient 2019

Figure 2

All patient contacts in DPV within the entire years 2019 and 2020. Data lines were smoothed using the LOESS-method. In 2019, typical seasonal effects during summer holidays (weeks 30-34) and at turn of the year reduced outpatient contacts (red, dashed line). In 2020 COVID19-lockdown had a strong nonseasonal impact (red solid line). Obvious lockdown effects were also decreased inpatient admissions and increased telemedicine use (blue and green lines). Telemedicine dynamics is depicted exclusively in Figure $\mathrm{S} 2$ in supplemental information. 


\section{Supplementary Files}

This is a list of supplementary files associated with this preprint. Click to download.

- Supplementaryinformation.docx 